\title{
Evacuation Route Calculation of Inner Buildings
}

\author{
Shi Pu and Sisi Zlatanova \\ Delft University of Technology, OTB Research Institute for Housing, \\ Urban and Mobility Studies, Jaffalaan 9, 2628 BX Delft, the Netherlands. \\ Email: s.pu@ewi.tudelft.nl; s.zlatanova@otb.tudelft.nl
}

\begin{abstract}
Disastrous accidents (fire, chemical releases, earthquake, terrorist attacks, etc) in large public and residential buildings (discotheques, cafes, trade and industrial buildings) usually result in tragic consequences for people and environments. Such accidents have clearly showed that need for reliable systems supporting rescue operations is urgently appealing. Amongst all, giving appropriate information to the ordinary people in/around the affected area considering the disaster developments (available exists, assessable corridors, etc.) and the human factors (age, gender, disability) are of critical importance for the success of the rescue operation.

This paper promotes a new approach (based on 3D models) for giving evacuation instruction to people. The paper is organised in three general parts. The first part discusses briefly current approaches for alarming people in buildings showing their disadvantages. The second part presents the 3D system architecture and elaborates on the needed components. Discussion on the required developments concludes the paper.
\end{abstract}

\section{Introduction}

Since the 'birth' of buildings, the damage from both natural and unnatural disasters never stops. Current buildings are designed higher and more complex than ever before, therefore the potential disasters are also various. Generally, these disasters can be grouped into the following types (William H. Stringfield, 1996):

- Primary disasters such as: fire, power outage, terrorism (bombing incidents, bomb threat, taking of hostages, etc.), chemical releases (radioactive materials, toxic gases, etc.), earthquake, flood, hurricanes, etc. 
- Secondary disasters. For example, an earthquake could cause a structural fire, which may in turn burn out circuits resulting in a power failure.

All of the above disasters require people inside buildings to be evacuated as soon as possible. Considering the complexity of modern buildings and the great numbers of people that can be inside buildings, it is rather difficult to organise a quick evacuation. Very often serious problems such as huddle, trample, inaccessibility of exits, etc are observed. Typical examples of such tragical circumstances are the fires in a cafe in Volendam, the Netherlands (2001) and discotheque in Brazil (2004).

In order to make minimal losses from these disasters, many evacuation strategies have been researched and some of them are widely accepted or even integrated into architecture design. This paper will focus on the importance of 3D geo-information. Geo-information has been widely used in all the Disaster Management Phases, e.g. Mitigation, Preparedness, Recovery, but hasn't been really applied to the Response phase (Zlatanova and Holweg 2004) and especially inside buildings. This paper gives a new concept of inner buildings evacuation strategy, which aims at developing of a knowledge-based system able to provide dynamic, specific and accurate evacuation guidance based on indoor geo-information, and sends these instructions to people with interactive instructions.

Next section introduces the evacuation strategies used in current buildings and discusses their drawbacks. After that the overall architecture of our concept model is presented with detailed explanations of the three main technical parts. This paper is closed with conclusion and recommendations for future research.

\section{Current Evacuation Systems}

A lot of developments and research are aiming at providing more efficient means for alarming and guiding people. Good examples are fire allariming systems. Many buildings are currently equipped with modern fire detection systems and it is possible to alert people to a fire. However, this gives no clues as to how to escape. Directional Sound Evacuation (DSE) beacons are also available and they can eventually give clear audible navigation to nearest exit (http://www.soundalert.com/dse_buildings.htm). Sevrallarge public buildings (Business Design Centre, London, Munich International Airport, etc.) are equipped already with DSE. These systems can be combined with sophisticated analogue addressable Fire Alarm Control Panels (FACP) (e.g. http://www.adt.co.uk/fire_panels.html). These are systems 
that can locate seats of fires and decide which are preferred evacuation routes. DSE can then be activated only along these routes. It is also possible to teach people what is the meaning of sounds. The problem is still that these kinds of systems react only on the seat of the fire and are 'blind' about situation after the fire alarm is triggered.

In principle, current alarming systems can be subdivided into three groups (Galea et al, 1999):

Automatic detection system involve a sensor network plus associated control and indicating equipment. Sensors may detect heat, smoke or radiation and it is usual for the control and indicating equipment to operate an alarm system. It may also perform other signaling or control functions, such as the operation of an automatic smoke control system.

Alarm system alert people at the early stages of a disaster and give them maximum escape time. Normally any of following devices needs to be incorporated in the building:

- Manually operated sounders;

- Simple manual call points combined with bell, battery and charger;

- Internal speed communication system (telephone, intercom, etc.) should be provided so that conversation is possible between every floor and the control centre.

Emergency lighting is designed to allow occupant to continue to occupy, although they may not operate as efficiently as under normal lighting.

Additionally, building construction requirements to resist potential natural and industrial disasters exist (that may vary from country to country). Evacuation exits should satisfy certain criteria so that people can pass through the exits safely and quickly. Different standards give different requirement for exits' width, numbers, capacities, etc. Generally, there should be:

- 1 exit for up to 60 persons;

- 2 exits for 61-600 persons;

- 3 exits for over 600 persons.

Despite the variety of systems, current emergency alarming systems are only able to provide simple mostly constant evacuation instructions (evacuation plans, green lights, sounds, voice) which is not sufficient because:

- Evacuation plans lack flexibility. Evacuation notifications usually directly follow pre-defined evacuation plans, regardless whatever has happened or is happening in buildings. This may lead people into dead ends (collapsed ceilings, destroyed stairs, blocked exits, etc.) or bring 
more serious problems (lead to a spaces with gas leakage and possible explosions).

- Evacuation plans is not intelligent enough. If not controlled, too many people rush to same exits, which results in the 'traffic jam' in inner buildings, and would greatly delay the speed of evacuation, may results in blockage of exits and many injured people.

- Evacuation instructions give insufficient information. For people who are not familiar with the building, current evacuation instructions may not be helpful of even of no use. Some serious disasters (power outage, fire, etc.) that reduce visibility can make their situations even worse.

The problems mentioned above are result of three serious deficiencies:

- Lack of indoor geo-information (about the structure of the building),

- Lack of dynamic information (about the current situation),

- Lack of flexible means for evacuation instructions.

A more elaborated systems are needed that can offer intelligent, knowledge-based evacuation navigation. How such a system should react? Let's assume that a fire accident happened on the $10^{\text {th }}$ floor of a 21 -floor building, and expanded to the $9^{\text {th }}$ and $11^{\text {th }}$ floor in a very short period. People in this building should be evacuated immediately! In a sequence of scenes we will give a possible development of the situation:

- Scene 1. Evacuation lights were turned on, indicating the direction of evacuation exits.

- Scene 2. Voice instructions sounded from the speakers all over the building, asking people below $9^{\text {th }}$ floor to evacuate from Exit A, people on $9^{\text {th }}, 10^{\text {th }}, 11^{\text {th }}, 12^{\text {th }}, 13^{\text {th }}, 14^{\text {th }}, 15^{\text {th }}$ floor to evacuate from Exit B, people on $15^{\text {th }}, 16^{\text {th }}, 17^{\text {th }}$ floor to evacuate from Exit $C$, and people above $17^{\text {th }}$ floor to evacuate from Exit D. Never use elevators!

- Scene 3. Joanne was visiting this building when the accident happened. She was unfamiliar with the building, and she easily found herself totally lost in the building because the power system went down. But she had her PDA and suddenly she noticed that it is blinking. She opened it and realized that she receives instructions hoe to get out of the building. She followed the interactive graphical instructions and finally evacuated safely from the building.

- Scene 4. Andy and his colleagues were in the $11^{\text {th }}$ floor when the accident happened. Following the voice instructions, they went to Exit B immediately. But shortly after that they found the stairs of Exit B, $9^{\text {th }}$ floor was totally in fire! 'Where shall we go?' they asked themselves. Some of the group prefer to go to Exit A because it was the nearest. But Andy said 'NO!' because according to his PDA, most parts of Exit A had lost power. If they go to Exit A, they might be lost in total darkness! 
According to the instructions on the PDA, the best route was to go back to the $10^{\text {th }}$ floor, go to Exit $\mathrm{D}$, and then go down to the ground floor. And this is what exactly they did.

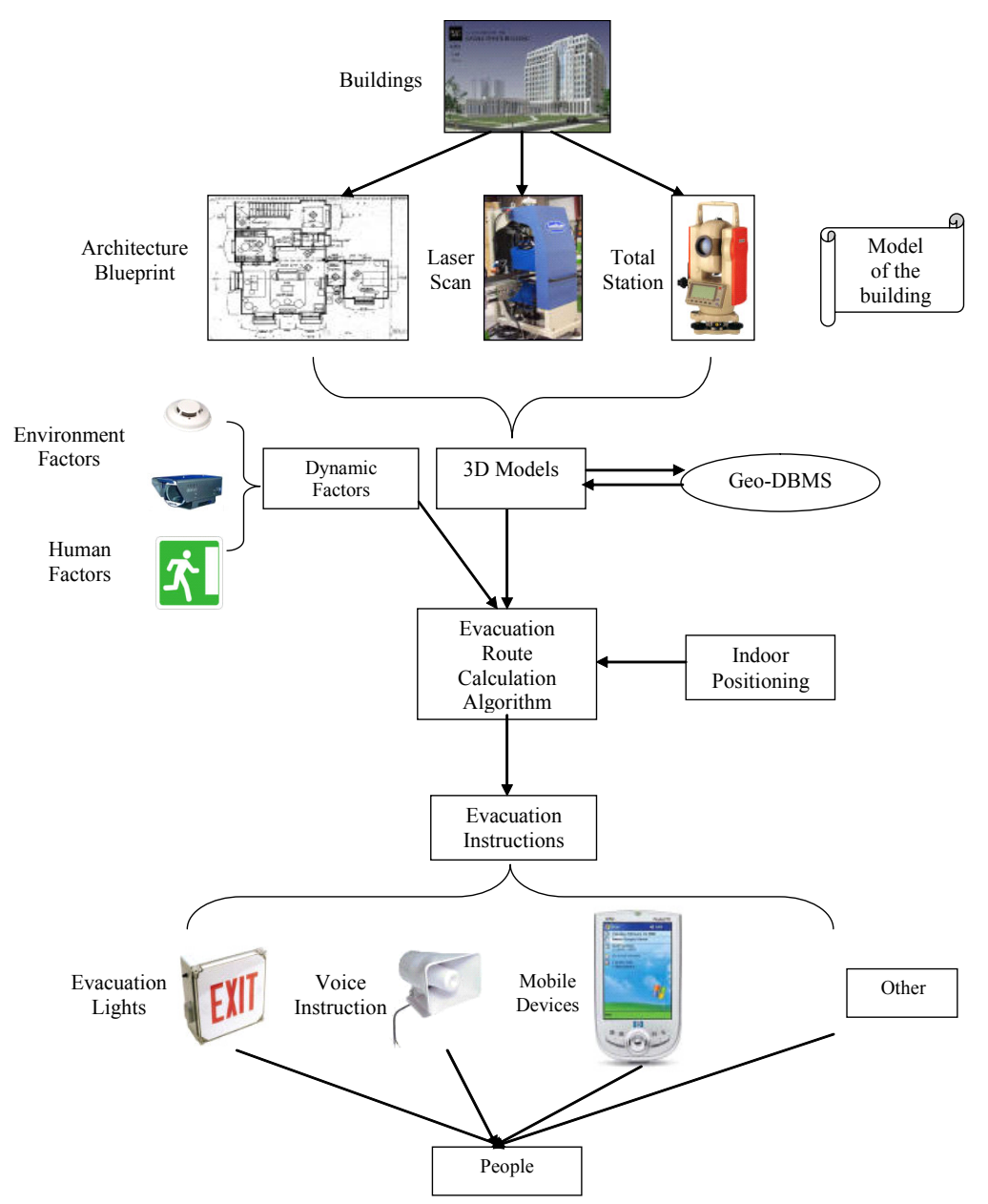

Fig. 1. Overall architecture of evacuation route calculation system

\section{A System for an Evacuation Route Calculation}

To be able to provide such a dynamic navigation four important components are necessary: system for indoor positioning, communication connection, inner model of the building, information about the people in the 
building, real-time information about the disaster, engine for route calculation (Figure 1) and engine for adapting the routing presentation.

Presently there're many techniques for positioning (passive and active), for example, the Global Positioning System (GPS), digital compass, and etc. However, most of these positioning techniques are only for outdoor positioning. In the recent couple of years, many indoor positioning techniques have been suggested (Gillieron and Mrminod, 2004, Kaemarungsi and Krishnamurthy, 2004, Fritsch et al 2001) using WLAN, location fingerprinting, vision system or combinations of them. For example, using location fingerprinting, one first collects the local fingerprints by performing a site-survey of the Received Signal Strength (RSS) from multiple Access Points (APs). Then the APs sends the RSS measurements to a central server, so that certain algorithms can be used to determine the estimated position of the signal, or in other words, the position of the individual.

For simplicity, we will consider that 3D indoor positioning (passive or active) and a telecommunication network (WLAN, GPRS or UTMS) are available (see Zlatanova and Verbree, 2003) and will concentrate only on the $3 \mathrm{D}$ indoor model, the algorithms for route calculation and the presentation of the information.

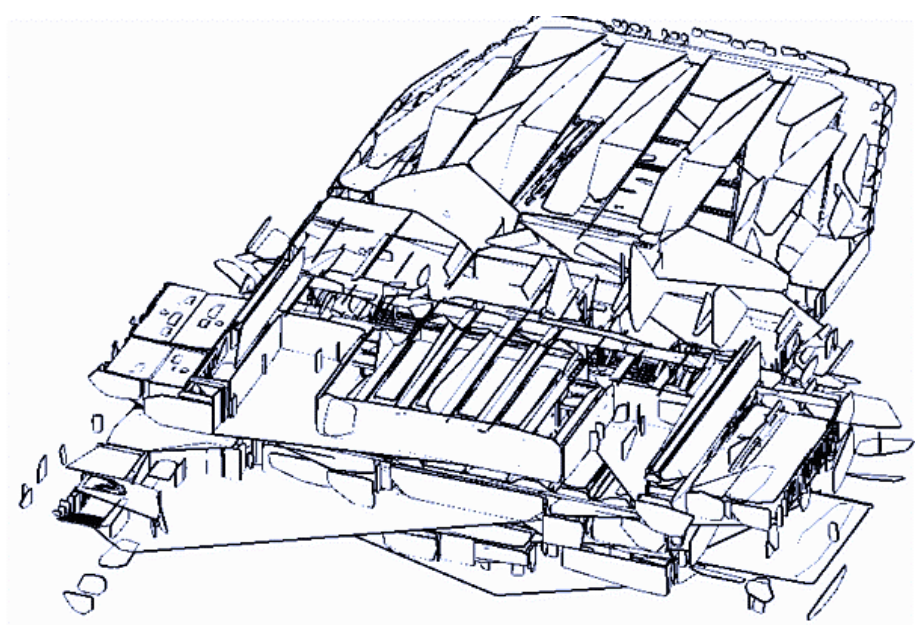

Fig. 2. Geometry of the Aula

\subsection{D Model of Buildings}

Modeling buildings will be understood here as the process of converting a building's architecture structure to a model that can be recognized by 
computer. With the help of this model, fastest and safest evacuation routes can be calculated both globally (for crowd) and locally (for a single person). The model of a building can be either a geometry/topology model, which means, an abstraction of a building is represented with polygons in 3D space (see also Zlatanova et al, 2004); or a logical model (see Lee, 2001), which represents the connections between the rooms. The rooms and important crossings are represented with nodes; the paths are represented as links between nodes.

Geometry model. Building a geometry model of interiors of buildings is more complex compared to outside model. The interior structure has to be seen as an aggregation of several different types of objects (rooms, stairs, etc.) with different shape. Geometry model (e.g., Figure 2) of a building can be generated in many different ways:

- Architecture blueprint of this building

- From point clouds obtained from terrestrial laser scanning

- Close-range images

- Surveying measurements

Each of the methods has advantages and disadvantages. Architectural blue prints are used very often for 3D models. Given the height of the floors, the 3D model can be manually 're-designed' in appropriate modeling software, e.g. 3D StudioMax, AutoCAD, MicroStation, etc. The common bottleneck is that the plans are either not available or differ from the real situation.

Laser scanning is relatively easy but results in a large amount of data (many of them even not necessary for the application, e.g. curtains, tables, etc) that has to be further filtered and modeled to obtain the 3D polygons. In general the human eye can easily understand geometry model and get an overview of a building out of point clouds. However the computer does not distinguish between the points. Therefore object recognition is needed. 3D building reconstruction from point clouds requires determination of rooms, corridors, stairs, etc. Different commercial software (e.g. Cyclone, CloudWorx) exists that allows a primitive (plane, cone, cylinder, etc.) to be fit to a group of points. The fitted element can be further aggregated into objects. This procedure is mostly manual and rather time consuming. Research towards automatic fitting procedures is currently going0n (e.g. Rabani and Van den Heuvel 2004), but still complete automation is not possible.

3D reconstruction using close-range images (taken with conventional camera) allows the operator to select only the points that are needed for the model (e.g. using PhotoModeler). The reconstruction is again in large amount manual (pointing the same points in different images and connect- 
ing selected points into triangles and polygons), but thanks to the images the operator orients better (compared to point clouds) in the model. Closerange images are wisely used for $3 \mathrm{D}$ reconstruction of historical buildings.

Surveying, in contrast to laser scanning and similar to close-range photogrammetry provides the points only needed for the modeling. To be able to reconstruct for example a simple room of four walls, one would need to measure only four points. Since the measurements are outside the office (in the building), a very good organization of the registered points is needed. Missed important points or unclear identification of points may lead to repeating of measurement. The actual $3 \mathrm{D}$ reconstruction is again mostly manual and rather time consuming.

There is not an automatic approach for $3 \mathrm{D}$ reconstructing of the interior of buildings and other closed constructions, and it is difficult to extract paths from geometry model. However, the 3D geometry is needed for presentation of the navigation route.

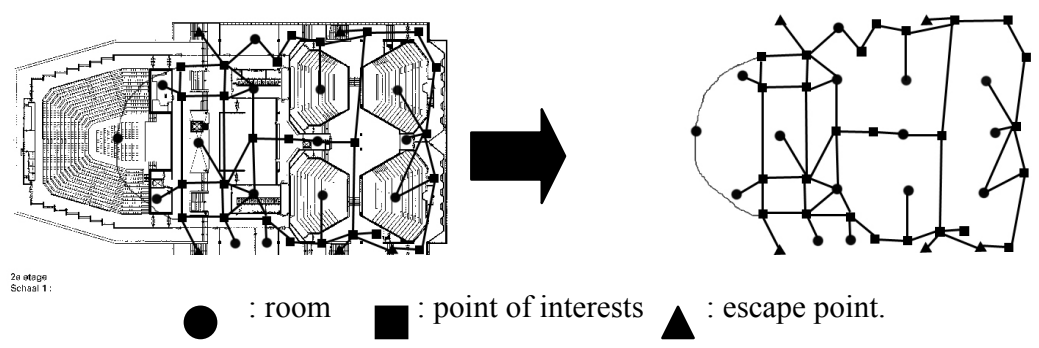

Fig. 3. Simplified Logical model of Aula, 2nd floor

Logical model. Logical model represents each room/crossing/exit in buildings with a node, and represents paths with the links between nodes. Using logical model, the inner structures of buildings instead of geometric information can be analyzed, and routes from node to node can be calculated easily, which is the most significant advantage over the geometry model (Gillieron and Merminod, 2004).

However, it is very difficult to generate the logical model (Figure 3 ) of a building automatically from its geometry model. We have to manually or half manually (with computer aided applications) create the important nodes and make their links. Especially, the links should be assigned with cost values, to represent the travel distance or travel time for each links. Search algorithms will consider the links' cost values and non-spatial data of nodes (will be discussed later) together, and then determine the most appropriate evacuation routes.

A lot of attention and experience are required to analyze the building and determine the nodes for logical model. This looks a tedious job, but 
once the logical model is completed, path search within the logical model can be easily done because this have been researched many years and there're already a number of well-developed search algorithms now which can be used directly to calculate the evacuate route. However, logical model can't be visualized directly by people as the geometry model does.

Hybrid model. A combination of geometry model and logical model can become a powerful tool for evacuation route calculation. The logical model will be used to compute the shores available path and the geometry model will be used for visualization. With links between these two models, information can be shared so that any route in logical mode can be reflected in geometry model.

Such combination has been implemented by Oracle and published as the Network Data Model in Oracle Database 10g. There're three kinds of Network Data Models: a logical network (no geometry), a geometry network (has geometry) and a LRS geometry network (has geometry with measure value). All of these networks can be hierarchical. They are all made up of four tables:

- Node table (Table 1). Stores the nodes, columns of this table include id, name, type, partition, cost, and etc.

- Link table (Table 2). Stores links between nodes, columns of this table include link id, name, type, start node, end node, cost (can be used to represent length), and etc.

- Path table

- Path link table

\begin{tabular}{lll}
\hline Name & Type & Example \\
\hline NODE_ID & NUMBER & 3 \\
NODE_NAME & VARCHAR2(200) & 'Meeting Room A' \\
NODE_TYPE & VARCHAR2(200) & 'Room' \\
ACTIVE & VARCHAR2(1) & 'Y' \\
PARTITION_ID & NUMBER & 2 \\
& & MDSYS.SDO_GEOMETRY \\
& & $(2003$, NULL, NULL, \\
GEOMETRY & MDSYS.SDO_GEOMETRY & SDO_ELEM_INFO_ARRAY \\
& & $1003,3)$, \\
& & SDO_ORDINATE_ARRAY \\
& & $(100,200,500,700))$ \\
\hline
\end{tabular}

Table 1 Columns of Node table

\begin{tabular}{lll}
\hline Name & Type & Example \\
\hline LINK_ID & NUMBER & 2 \\
\hline
\end{tabular}


$1152 \mathrm{Pu}$ Shi and Sisi Zlatanova

\begin{tabular}{|c|c|c|}
\hline LINK_NAME & VARCHAR2(200) & 'Corridor 2' \\
\hline START_NODE_ID & NUMBER & 3 \\
\hline END_NODE_ID & NUMBER & 5 \\
\hline LINK_TYPE & VARCHAR2(200) & 'Corridor' \\
\hline ACTIVE & VARCHAR2(1) & ' $\mathrm{Y}$ ' \\
\hline LINK_LEVEL & NUMBER & 1 \\
\hline GEOMETRY & MDSYS.SDO_GEOMETRY & $\begin{array}{l}\text { MDSYS.SDO_GEOMETRY } \\
\text { (2003, NULL, NULL, } \\
\text { SDO_ELEM_INFO_ARRAY } \\
\text { 1003,3), } \\
\text { SDO_ORDINATE_ARRAY } \\
(500,2100,550,3500))\end{array}$ \\
\hline COST & NUMBER & 6 \\
\hline
\end{tabular}

Table 2 Columns of Link table

Path table and path link table are optional, but calculated shortest path can be stored here. Networks can be created by either PL/SQL or Java API, but can only be analyzed by Java API. A shortest path function is already available in the Java API, but only the costs (length) of links are considered. Based on the existing well-structured network model, we can easily implement user-defined java functions that take other problems that affect the evacuation route calculation into consideration.

Non-spatial information. All the three models above organize the spatial information of inner buildings well. However, the model of a building with only spatial information is just an abstraction, which is not able to give the whole pictures for disasters in buildings. A complete evacuation model should also contain non-spatial information that influences the evacuation route. Some kinds of important non-spatial information are:

- Type of the spaces. Types for nodes (logical model) can be room, crossing, exit points and etc; types for links (logical model) can be corridor, stairs, and etc. Different types of element/node have different purposes and attributes. For example, the exit nodes are the target nodes for search algorithms; people have different moving speed in rooms, corridors, stairs, and etc.

- Population density. There're always more people in certain areas and less in other areas. For examples depending on the type of the building, in one room can be more than 40 and up to 200 people (theatres, congress centres) or less than two (offices). The system has to have information about the 'daily' distribution of people in the building

- Construction type. Different types of buildings have different requirement for the evacuation routes. For example, 'safe' is more important than 'fast' for evacuation routes in hospitals; evacuation route calcula- 
tion should be more careful about capacities of rooms in congress centres and theatres. Importance of different factors should also be adjusted a bit in order to be more suitable for different types of constructions.

- Type of utilities/networks. There might be potential dangerous or helpful utilities in certain areas. For example, gas that can explode, high voltage devices, telecommunication networks, medical utilities, and etc. These kinds of non-spatial information should not be ignored because they also have rather large influences on the evacuation routes calculation.

\subsection{Dynamic Factors}

Emergency situations are not static, and route calculation cannot only consider travel distance. There're many other factors that would affect the optimal evacuation routes. Theses factors can be grouped into two kinds:

Environmental factors. Unknown problems can happen to a building at any time during a disaster. The evacuation routes calculated from fixed search tree can be of no use at all, or even give wrong evacuation instructions. In such cases, an adaptive search tree, which reflects the real time status, can be of great help to make valid and accurate evacuation routes. Modern buildings are equipped with detection system that is able to detect some kinds of disasters such as fire/smoke/etc. The detection system can be connected to the buildings' logical models to form the adaptive search trees. Once a disaster is detected by sensors or showed from monitors, the nodes within dangerous areas should be marked automatically or manually as 'unsafe' or 'inaccessible' in the non-spatial information of a building's logical model. Then an evacuation route, which avoids the dangerous areas, should be calculated from current search tree. If more events are detected later, then the evacuation routes should be recalculated and the modified evacuation instructions should be sent to the affected people, if the new evacuation routes are different from the previous ones.

Different types of dynamic factors may be defined. An initial classification follows:

- Damage status. Areas that are already damaged should be avoided in an evacuation route. The damage status of an element (geometry model) or a node (logical model) can be True or False.

- Toxicity status. Areas that are already full of toxic gases/smokes should be avoided in an evacuation route. The toxicity status of an element (geometry model) or a node (logical model) can be True or False. 
- Power status. Places that lost power should not be in an evacuation route. The power status of an element (geometry model) or a node (logical model) can be True or False.

- Capacity of the routes. If people are given an evacuation route that did not consider the capacities of routes, they would probably get stuck in some low-capacity parts. This is quite dangerous, and should be avoided with no doubt. Evacuating from multi routes will efficiently avoid evacuation jams. Calculation of multi route evacuation will be discussed later in this chapter.

Human factors. There has been a lot of research on human behavior under different situations. From these researches, people's speed of movement varies with:

- Population density. If the density is higher than a certain value (crowddensity value), conditions would become very uncomfortable and movement is difficult or impossible. Nelson and Maclennan's research (quoted in Galea et al 1999) on human behaviour suggests that:

$\circ$ If population density $<0.54 \mathrm{p} / \mathrm{m}^{\wedge} 2$, individuals maintain movement speed.

$\circ$ If population density $>3.8 \mathrm{p} / \mathrm{m}^{\wedge} 2$, there is no or very little movement.

o Between these limits, speed is hindered and is a function of the density given by:

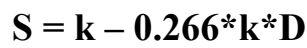

where ' $S$ ' is the practical movement speed $(\mathrm{m} / \mathrm{s})$; ' $\mathrm{D}$ ' is the population density $\left(\mathrm{p} / \mathrm{m}^{\wedge} 2\right)$; ' $\mathrm{k}$ ' is the standard movement speed. ' $\mathrm{k}$ ' is dependent on type of terrain, for example, $\mathrm{k}$ for corridors, doorways and ramps are 1.4.

- Age and gender. Ando's research on crowd movement can be summarized as: (quoted in Galea et al 1999):

○ Walk speed peaked at about 20 years of age.

- Males outpaced females at all ages.

- Max walk speed for males is about $1.6 \mathrm{~m} / \mathrm{s}$.

- Max walk speed for females is about $1.4 \mathrm{~m} / \mathrm{s}$.

- Level of disability. Problems for disabled people are not only in slowed down movement speed. People with specific movement aids, such as wheelchairs, cannot use stairs unaided. An evacuation flow with disabled people could have lower movement speed because normal occupants would help them along the way.

- Terrain effects. People move slower in certain areas, e.g. stairs. Fruin's research (quoted in Galea et al 1999) on people's behavior on stairs shows that the default stair travel speed are: 


\begin{tabular}{llll}
\hline Gender & Age & Down average $(\mathbf{m} / \mathbf{s})$ & Up average $(\mathbf{m} / \mathbf{s})$ \\
\hline Male & $<30$ & 1.01 & 0.67 \\
Female & & 0.755 & 0.653 \\
Male & $30-50$ & 0.86 & 0.63 \\
Female & & 0.665 & 0.59 \\
Male & $>50$ & 0.67 & 0.51 \\
Female & & 0.595 & 0.485 \\
\hline
\end{tabular}

Table 3 Fruin's research result about movement rates on stairs

The environment and human factors above will be both considered for the evacuation route calculation. Their importance may vary in different kinds of buildings. For example, population density should be carefully considered in theaters, level of disability should be carefully considered in hospitals, and etc. Values for these factors can be collected from investigation over the buildings. Many commonly used investigation methods are mentioned in Galea et al 1999. All the factor values should be stored along with the logical model as non-spatial information in the database.

\subsection{Route Calculation Algorithm}

Below we give the algorithm for evacuation calculation. It takes a building's model and dynamic factors as parameter, and aims at giving correct and safe evacuation routes, which will be sent to people with different evacuation instructions.

\subsubsection{Basic Route Calculation}

With the logical model of a building, routes from a certain node to another node can be calculated using a lot of existing search algorithms, if only the travel distances between nodes are taken into consideration.

A search algorithm is generally evaluated from four criteria (Russel and Norvig, 1995):

- Completeness: is the algorithm guaranteed to find a solution when there is one?

- Time complexity: how long does it take to find a solution?

- Space complexity: how much memory does it need to perform the search?

- Optimality: Does the strategy find the highest-quality solution when there are several different solutions? 
Among these four criteria, completeness is not critical because we only need the best route (optimality) not every route, with acceptable computation time and memory requirement. Below we just give the definitions and evaluation criteria of four popular search algorithms. More detailed information and proof of the evaluation criteria can be found in the literature (see Russel and Norvig, 1995).

Breadth-first search In breadth-first search, the root node is expanded first, then all the nodes generated by the root node are expanded next, and then their successors, and so on, till find the goal node.

Depth-first search Depth-first search always expands one of the nodes at the deepest level of the tree. Only when the search hits a dead end does the search go back and expand nodes at shallower levels. The drawback of depth-first search is that it can get stuck going down the wrong path.

Depth-limited search Depth-limited search avoids the pitfalls of depthfirst search by imposing a cut off on the maximum depth of a path.

Iterative deepening search Iterative deepening search is a strategy that sidesteps the issue of choosing the best depth limit by trying all possible depth limits.

Bidirectional search Bidirectional search algorithm searches both forward from the initial state and backward from the goal, and stop when the two searches meet in the middle.

Dijkstra's algorithm Dijkstra's algorithm differs from the first five in that it is an informed search algorithm, which means the 'goodness' of a node can be estimated. This algorithm keeps two sets of vertices Stringfield, 1996:

- bbS: the set of vertices whose shortest paths from the source have already been determined

- bbV-S: the remaining vertices. The other data structures needed are:

- d: array of best estimates of shortest path to each vertex.

- pi: an array of predecessors for each vertex. The main steps of Dijkstra's algorithm are:

- Initialise $\mathbf{d}$ and $\mathbf{p i}$,

- Set $\mathbf{S}$ to empty,

- While there are still vertices in $\mathbf{V}-\mathbf{S}$,

- Sort the vertices in $\mathbf{V}-\mathbf{S}$ according to the current best estimate of their distance from the source,

- Add $\mathbf{u}$, the closest vertex in $\mathbf{V}-\mathbf{S}$, to $\mathbf{S}$,

- Relax all the vertices still in $\mathbf{V}-\mathbf{S}$ connected to $\mathbf{u}$. The relaxation process updates the costs of all the vertices, $\mathbf{v}$, connected to a vertex, $\mathbf{u}$, if we 
could improve the best estimate of the shortest path to $\mathbf{v}$ by including $(\mathbf{u}, \mathbf{v})$ in the path to $\mathbf{v}$.

Table 4 compares the first five algorithms in term of the four criteria.

\begin{tabular}{llllll}
\hline Criteria & $\begin{array}{l}\text { Breadth- } \\
\text { First }\end{array}$ & Depth-First & $\begin{array}{l}\text { Depth- } \\
\text { limited }\end{array}$ & $\begin{array}{l}\text { Iterative } \\
\text { Deepening }\end{array}$ & $\begin{array}{l}\text { Bidirec- } \\
\text { tional }\end{array}$ \\
\hline Time & $b^{d}$ & $b^{m}$ & $b^{l}$ & $b^{d}$ & $b^{d / 2}$ \\
Space & $b^{d}$ & $\mathrm{bm}$ & $\mathrm{bl}$ & $\mathrm{bd}$ & $b^{d / 2}$ \\
Optimal & Yes & No & No & Yes & Yes \\
Complete & Yes & No & Y, if 1 $\geq \mathrm{d}$ & Yes & Yes \\
& & & &
\end{tabular}

Table 4 Evaluation of search algorithms. $b$ is the branching factor ${ }^{1} ; d$ is the depth of solution, $m$ is the maximum depth of the search tree; 1 is the depth limit (Russel and Norvig, 1995)

Normally there're several hundreds of nodes in logical models of modern buildings. Experiments show that all of the above search algorithms take approximately 1 second to find all the solutions (if any) for trees with hundreds of nodes. Therefore here it doesn't matters too much about which algorithms to use.

\subsubsection{Advanced (Evacuation) Route Calculation}

Basic search algorithms can only be used to calculate the routes, but not evacuation routes. During a disaster in a building, the route network may change at any time. Therefore we need an advanced algorithm that also considers dynamic factors as discussed in Section 3.2. If not only the cost of links, but also the dynamic environment factors and human factors are taken consideration, then the earlier basic route calculation algorithms should be improved in several aspects:

- Try to avoid traffic jams. Traffic jams are the result of either too high population density or small capacity of routes. People should be evacuated to exits from different routes, which have approximately same population densities.

- Adaptive to real time status. Once the status of some nodes are changed, for example, from 'normal' to 'dangerous', then the calculation procedure should be repeated using the current model.

- Give special evacuation routes for disabled people. If applicable, evacuation elevators are the first choice for disabled people. If not, dis-

1 If in a hypothetical tree where every nodes is connected to b nodes, we say the branching factor of this search tree is $b$. 
abled people and their aid workers should be assigned to less used routes because their movement can significantly decrease the speed of exodus evacuation.

Here we propose an algorithm for evacuation route calculation, which aims at improving the basic route calculation algorithm in above three aspects.

- Build a search tree from the logical model. Specify each node with two estimated population density value: for disabled people, and for nondisabled people. Specify the global movement speed values for disabled people and non-disabled people. Specify the crowd-density value.

- Scan the current searching tree, remove the unsafe nodes, and build a new tree.

- Run the basic route calculation algorithm and find the evacuation route for people within each node. This will result in a time line of the evacuation.

- Split the time line into several time segments Ti.

- For a segment Ti in time order, calculate the current population densities for each node. The current population density $\mathrm{D}$ is:

$$
D=D_{n}+D_{d} \times m
$$

where $D_{n}$ is the population density of non-disabled people; $D_{d}$ is the population density of disabled people; $\mathrm{m}$ is a constant number which is larger than 1 .

If the current population density $\mathrm{D}$ is higher than the crowd-density value or the route capacity of this node, go to 6 , else go to 8 .

- Go back to the previous time segment (i-1), reallocate the flows which 'will' join in the jam nodes to other routes. Try different allocation till no traffic jam will happen. Notice that people from same initial nodes should be allocated to the same route.

- Step into the next time segment, and repeat 5-7, until finish the last time segment.

- Evacuation route calculation completed.

This algorithm is to be implemented in a hybrid model as described in Section 3.1. Tests to be performed on the model of the Aula (Figure 3) will valid the algorithm and will supply knowledge for further improvements. Expectations for improvement are given in the later in this paper.

\subsection{Evacuation Instructions}

Once the route is calculated, a very important question is how to represent it to the user. There're many possibilities (depending on the available tech- 
nology) to give evacuation instructions from calculated evacuation routes (Figure 4).
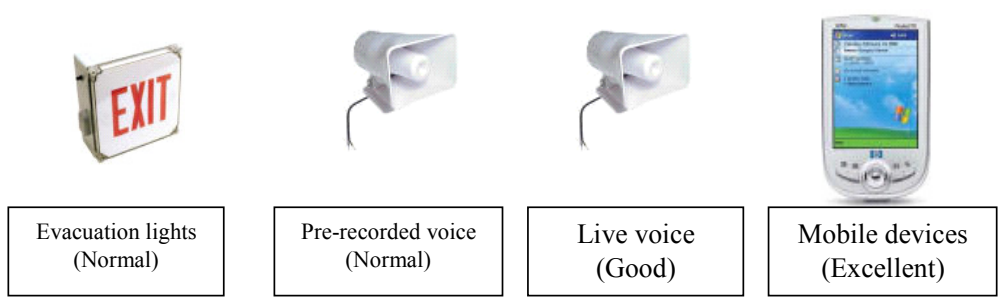

Fig. 4. Effectiveness of different evacuation routes

Evacuation lights and speakers can be used to give evacuation instructions for all the people within a building. Voice usually gives helpful information, but it is still difficult to generate voice instructions automatically from calculated evacuation route. A possible solution is to first generate the path figures that can be easily understood by administrators, so that they can give use microphones to live evacuation instructions.

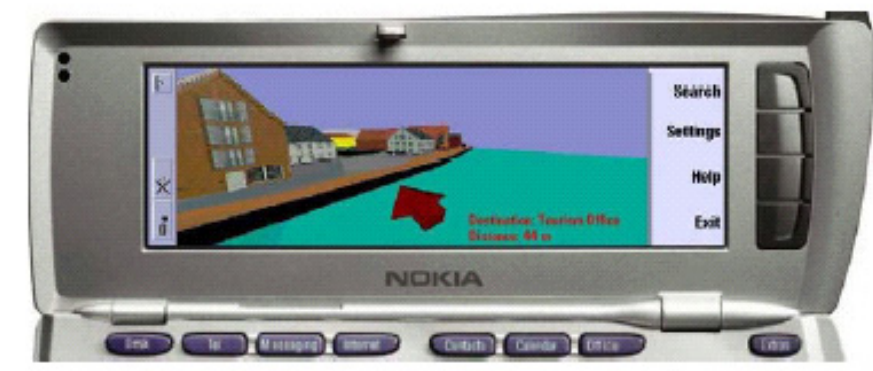

Fig. 5. 3D models with Nokia GL on the Communicator 9210 as one result of the TellMaris project (IST-2000-28249, http://www.tellmaris.com). C) Nokia Research

The most advances possibility is to use mobile devices like cell phone, PDA, etc. Mobile devices can supply people with interactive and graphical evacuation instructions. Using 3D location-based services, the received evacuation routes can be always adapted to the real time position, so that people don't need to remember all the evacuation details. Another advantage of using mobile devices is the graphical instruction. A large number of mobile devices are able to show pictures or videos, some even embedded with 3D rendering engines (Figure 5). Evacuation instructions can be sent from central computer to mobile terminals with either video streams or evacuation directions, or combination of both. Video streams are al- 
ready rendered in the server from both the geometry model and evacuation directions (from logical model). This method has low requirement for the mobile terminals because they only need to display the videos, but the amount of transferred data is rather considerable. If only evacuation directions are sent via wireless network, the transferred data is not heavy, however, mobile devices need to render the visualization locally, which might be tedious task for slow mobile devices. Balanced visualization between sending video frames and evacuation directions can lead to satisfactory results.

\section{Concluding Remarks}

This paper suggested a new concept of evacuation route calculation for inner buildings using 3D models and considering human and environment factors. The 3D model (hybrid model) will be organised in DBMS. The evacuation algorithm is based on the basic search algorithms, but it also takes the timeline of an evacuation into consideration. The calculated routes could be sent presented in various ways so that they could reach as many people as possible. Mobile devices like PDA can be very helpful to receive evacuation instructions.

Based on the concepts given in this paper, implementation of the evacuation calculation algorithm and further research will be carried on. Accuracy and speed are certainly the two most important evaluation parameters for the system. It might be also necessary to give multiple evacuation routes to users so that they can have more possibilities to choose. We consider focussing on the following aspects:

- Determine exactly which kinds of environment factors and human factors should be considered, and how can they be organized as non-spatial information in the Geo-DBMS.

- Create an automatic procedure for linking logical model with geometry model.

- How to make more accurate timeline for the evacuation process?

- How can this system be technically fast and robust enough to really be useful in a disaster? And how to validate the calculated evacuation routes?

- What is the balance between bandwidth and rendering capability for evacuation instructions via mobile devices?

- How can the system be modularized and gradually evolved? Since this algorithm aims at giving route instructions for inner buildings in disaster situations, it is also possible to give route guidance for normal situa- 
tions. It would be interesting to know how to evolve this algorithm to a guide system for inner buildings.

\section{References}

Buchana AH (2001) Structural design for fire safety, Wiley

Galea ER, Owen M, Gwynne S (1999) Principles and practice of evacuation modeling second edition, CMS Press

Gillieron P, Merminod B (2004) Personal navigation system for indoor applications, $11^{\text {th }}$ IAIN World Congress

Fritsch D, Klinec D, Volz S (2001) NEXUS---positioning and data management concepts for location-aware applications, Pergamon, Computers, Environment and Urban Systems 25, pp 279-291

Kaemarungsi K, Krishnamurthy P (2004) Modeling of Indoor Positioning Systems Based on Location Fingerprinting, IEEE INFOCOM

Lee J (2001) 3D Data Model for Representing Topological Relations of Urban Features', Proceedings of the $21^{\text {st }}$ Annual ESRI International User Conference, San Diego, CA, USA

Morris J (1998) Data Structures and Algorithms. Available at http://ciips.ee.uwa.edu.au/ morris/Year2/PLDS210/dijkstra.html

Oracle (2003) The Network Data Model manual of Oracle Database 10g, Oracle

Rabbani T, Van den Heuvel, F (2004) 3D industrial reconstruction by fitting CSG models to a combination of images and point clouds, IAPRS vol XXXV part B5, Istanbul, pp 7-15

Russel, Norvig (1995) Artificial intelligence a modern approach first edition, Prentice Hall

Stringfield WH (1996) Emergency planning and management, Rockville: Government Institutes

Zlatanova S, Holweg D, Coors V (2004) Geometrical and topological models for real-time GIS, Proceedings of UDMS 2004, 27-29 October, Chioggia, Italy, CDROM, $10 \mathrm{p}$

Zlatanova S, Holweg D (2004) 3D Geo-information in emergency response: a framework, Proceedings of the 4th International Symposium on Mobile Mapping Technology (MMT'2004), March 29-31, Kunming, China 6 p

Zlatanova S, Verbree E (2003) Technological developments within 3D locationbased services, International Symposium and Exhibition on Geoinformation 2003 (invited paper), 13-14 October, Shah Alam, Malaysia, pp 153-160 
